\section{Sur la dérivée faible d'un ensemble de fonctionnelles linéaires}

\author{
par .
}

\section{S. MAZURKIEWICZ (Varsovie).}

Soit $X$ un champ vectoriel de type $\left.B,{ }^{1}\right)$ séparable. Une suite de fonctionnelles linéaires $\left\{f_{n}(x)\right\}$ definies dans $X$ est dite faiblement convergente vers une fonctionnelle $f(x)$, si l'on a pour tout $x \& X$

$$
\lim _{n \rightarrow \infty} f_{n}(x)=f(x),
$$

ce que nous écrirons simplement

$$
\lim _{n \rightarrow \infty} f_{n}=f
$$

$f$ est alors une fonctionnelle linéaire ${ }^{2}$ ).

Nous dirons ${ }^{3}$ ) que la fonctionnelle $f$ est une fonctionnellelimile faible d'un ensemble $R$ de fonctionnelles linéaires, s'il existe une suite $\left\{f_{n}\right\}$ telle que $f_{n} \varepsilon R, f_{n} \neq f, \lim f_{n}=f$. L'ensemble $R^{\prime}$ de toutes les fonctionnelles-limites $\operatorname{de} \stackrel{n \rightarrow \infty}{R}$ sera appellé la dérivée faible de $R$. Nous dirons enfin que $R$ est faiblement fermé, si $R^{\prime} \subset R$.

On démontre facilement que $R^{\prime}$ peut ne pas être faiblement fermé. M. Ban ach a posé la question si cette circonstance peut se présenter lorsqu'on suppose en outre que $R$ est un ensemble linéaire ${ }^{4}$ ); je démontre dans cette Note que la réponse est affirmative.

\footnotetext{
1) C. à d. normé et complet.

3) S. Banach, Sur les opérations dans les ensembles abstraits, Fund. Math. III (1922) p. 157, Théorème 5 .

3) Ces notions sont dues à M. B an a ch.

4) $R$ est linéaire, s'il contient toute combinaison linéaire à coefficients réels de deux quelconques de ses fonctionnelles.
}

Considérons le champ $X$ de toutes les suites $\left\{\xi_{p}\right\}$ de nombres réels, convergentes vers 0 , et définissons la norme par la relation

$$
\left\|\left\{\xi_{p}\right\}\right\|=\operatorname{Max} .\left(\left|\xi_{1}\right|,\left|\xi_{2}\right| \ldots \ldots\right) .
$$

$X$ est un champ vectoriel de type $B$, séparable. Une fonctionnelle linéaire dans $X$ est de la forme

$$
f\left(\left\{\xi_{p}\right\}\right)=\sum_{p=1}^{\infty} a_{p} \xi_{p}
$$

les $a_{p}$ étant réels et la série $\sum_{p=1}^{\infty}\left|a_{p}\right|$ convergente. On vérifie facilement le lemme suivant:

Lemme. Soit

$$
\begin{aligned}
& f\left(\left\{\xi_{p}\right\}\right)=\sum_{p=1}^{\infty} a_{p} \xi_{p}, \\
& f_{n}\left(\left\{\xi_{p}\right\}\right)=\sum_{p=1}^{\infty} a_{p}^{(n)} \xi_{p} .
\end{aligned}
$$

Pour que $\left\{f_{n}\right\}$ converge faiblement vers $f$, il faut et il suffit que l'on ait

$$
\begin{gathered}
\lim _{p} a_{p}^{(n)}=a_{p}, \\
\varlimsup_{n \rightarrow \infty} \sum_{p=1}^{\infty}\left|a_{p}^{(n)}\right|<\infty .
\end{gathered}
$$

Rangeons maintenant en une suite infinie tous les couples de nombres naturels et désignons par $N(i, k)$ l'indice du couple $i, k$. Posons, pour $p$ naturel,

$$
\begin{aligned}
a_{i, k}^{(2 p-1)} & =\frac{1}{2^{p}} \text { pour } p \leqslant i \\
& =0 \text { pour } p>i \\
a_{i, k}^{(2 p)} & =i \text { pour } p=N(i, k), \\
& =0 \text { pour } p \neq N(i, k) .
\end{aligned}
$$

$$
f_{i, k}\left(\left\{\xi_{p}\right\}\right)=\sum_{p=1}^{\infty} a_{i, k}^{(p)}, \xi_{p}
$$

enfin soit $R$ l'ensemble de toutes les combinaisons linéaires finies 
des fonctionnelles $f_{i, k} . R$ est évidemment un ensemble linéaire. Nous montrerons que $R^{\prime}$ n'est pas faiblement fermé.

Posons, pour $p$ naturel,

$$
\begin{aligned}
a_{i}^{(2 p-1)} & =\frac{1}{2^{p}} \text { pour } p \leqslant i, \\
& =0 \text { pour } p>i, \\
a_{i}^{(2 p)} & =0, \\
a^{(2 p-1)} & =\frac{1}{2^{p}} \\
a^{(2 p)} & =0,
\end{aligned}
$$

et considérons les fonctionnelles

(7)

$$
\begin{gathered}
f_{i}\left(\left\{\xi_{p}\right\}\right)=\sum_{p=1}^{\infty} a_{i}^{(p)} \xi_{p}, \\
f\left(\left\{\xi_{p}\right\}\right)=\sum_{p=1}^{\infty} a^{(p)} \xi_{p} .
\end{gathered}
$$

D'après notre lemme, il résulte de (3), (4), (5), (6), (7)

$$
\begin{aligned}
& \lim _{k \rightarrow \infty} f_{i, k}=f_{i}, \\
& \lim _{i \rightarrow \infty} f_{i}=f,
\end{aligned}
$$

$$
\begin{aligned}
& A_{n}^{(2 p-1)}=\frac{1}{2^{p}} \sum_{i=1}^{\infty} \sum_{k=1}^{\infty} \alpha_{i k}^{(n)}, \\
& A_{n}^{(2 p)}=i \alpha_{i, k}^{(n)} \text { où } p=N(i, k) .
\end{aligned}
$$

D'après la condition (2) du lemme et d'après (6), (7), (10), (11) on aura

$$
\lim _{n \rightarrow \infty} \sum_{i=p}^{\infty} \sum_{k=1}^{\infty} \alpha_{i, k}^{(n)}=1
$$

D'autre part (11) entraîne

$$
\sum_{p=1}^{\infty}\left|A_{n}^{(p)}\right| \geqslant \sum_{p=1}^{\infty}\left|A_{n}^{(2 p)}\right|=\sum_{i=1}^{\infty} i \sum_{k=1}^{\infty}\left|\alpha_{i, k}^{(n)}\right| \geqslant q \sum_{i=q}^{\infty} \sum_{k=1}^{\infty} \alpha_{i, k}^{(n)}
$$

Donc on aura, d'après (12),

$$
\lim _{n \rightarrow \infty} \sum_{p=1}^{\infty}\left|A_{n}^{(p)}\right| \geqslant q
$$

quel que soit le nombre naturel $q$; mais cette relation est en contradiction avec la condition (2) du lemme.

donc $f$ est une fonctionnelle-limite faible de $R^{\prime}$; cependant $f$ n'est pas contenu dans $R^{\prime}$.

En effet, dans le cas contraire, $R$ contiendrait une suite $\left\{\varphi_{n}\right\}$ telle que $\lim _{n \rightarrow \infty} \varphi_{n}=f$.

On a

$$
\varphi_{n}=\sum_{i=1}^{\infty} \sum_{k=1}^{\infty} \alpha_{i, k}^{(n)} f_{i, k}
$$

où les $\alpha_{i, k}^{(n)}$ désignent des constantes réelles; pour une valeur donnée de $n$, il n-y-a qu'un nombre fini des $a_{i k}^{(n)}$ différents de 0 . Soit

$$
\varphi_{n}\left(\left\{\xi_{k}\right\}\right)=\sum_{p=1}^{\infty} A_{n}^{(p)} \xi_{p}
$$

d'après (3), (4), (9), (10) on aura 